Knipp Delores J. (Orcid ID: 0000-0002-2047-5754)

Hapgood Mike (Orcid ID: 0000-0002-0211-0241)

Welling Dan (Orcid ID: 0000-0002-0590-1022)

\title{
Maintaining a Strong Signal and Strong Impact
}

\author{
Delores Knipp, Mike Hapgood and Dan Welling \\ ${ }^{1}$ University of Colorado Boulder. \\ ${ }^{2}$ RAL Space, STFC Rutherford Appleton Laboratory, UK \\ ${ }^{3}$ University of Michigan.
}

Corresponding author (dknipp@agu.org)

Abstract: We discuss measures of the Space Weather Journal impact and influence

Space Weather Journal (SWE) is in its $14^{\text {th }}$ year. In the last five years submissions have increased $\sim 40 \%$ and published manuscripts have increased $\sim 20 \%$. The trend of our Journal Impact Factor (JIF), a widely used (and sometimes abused) measure of 'signal' in the publishing world, is upward. The JIF formula is below if you're not familiar with it. Ten years ago, SWE's first JIF was 1.6 (Lanzerotti, 2007). Thanks to the strong interest and contributions from space professionals, SWE's JIF has been on a long-term positive trajectory. This year SWE's 1-year and 5-year JIFs are both 2.6; a signal well above noise level in spite of a long 23-24 solar cycle minimum and less-than-spectacular solar cycle 24 maximum. (For more on journal metrics see: https://eos.org/editors-vox/journal-impact-factors-with-uncertainties)

$$
\text { SWE JIF }=\frac{\# 2016 \text { journal citations to SWE technical articles published in } 2014 \text { and } 2015}{\# \text { of published SWE technical articles in } 2014 \text { and } 2015}
$$

The JIF-approach to measuring impact is standardized across journals, but smaller science disciplines are at a slight disadvantage, and JIFfails to capture some of the quality factors that matter to authors (and editors). For example, the 2016 JIF didn't and couldn't capture that fact one of SWE's 2014 articles, Kelly et al. (2014), "Progress toward forecasting of space weather effects on UHF SATCOM after Operation Anaconda" (edited by L. Lanzerotti) was an American Association for the Advancement of Science (AAAS) "top 10 science story of the year." See http://www.sciencemag.org/news/2014/12/top10-science-news-stories-2014. SWE articles highlighted in Physics Today and other high-profile digests are similarly not counted as citations.

What other "impact" factors should a journal editor consider? International scope, breadth of topic, timeliness, and discoverability are high on our list. From its inception SWE has focused on international submissions since space weather impacts transcend national boundaries. Fifty percent of SWE submissions come from outside the US. Growing ground-based observational networks in China, Africa and South America are supporting this trend.

As noted in Lanzerotti (2007), "The papers in Space Weather attempt to build bridges between the science of space research and the applications of this research." To that end SWE has a healthy component $(\sim 20 \%)$ of feature, commentary and news article that keep readers informed about the hot

This is the author manuscript accepted for publication and has undergone full peer review but has not been through the copyediting, typesetting, pagination and proofreading process, which may lead to differences between this version and the Version of Record. Please cite this article as doi: $10.1002 / 2017$ SW001783 
topics in the space weather discipline and informs policy makers. During the last year SWE manuscripts have ranged from the details of radiation-measuring platforms in the atmosphere (Aplin et al., 2017) and at the International Space Station (Dachev et al., 2017), to a review of heliospheric imaging (Harrison et al., 2017), to scintillation of interplanetary spacecraft signals (Molera Calvés et al., 2017) to description and release of a decade-plus of in situ particle data from Global Positioning System satellites (Morley et al. 2017).

A hallmark of SWE has been rapid turn-around. Currently the typical time to first decision is about one month. This decision time has lengthened a bit as the editorial team has expanded to meet the demands of the 150+ submissions per year. Further, submitted manuscripts are becoming more complex with deeper data analysis from ground- and space-based instruments. Consistent with long-term vision, approximately $20 \%$ of SWE's published manuscripts in 2017 report on combinations of model-dataforecasting results. (As an excellent example see: Sharpe \& Murray (2017)). Acquiring reviewers for these involved topics from the busy pool of referees can be time-consuming. Our metric on manuscript turn-around indicates a high level of commitment on the part of the editorial team and SWE reviewers.

SWE strives to publish the best science, applications and review articles that maintain SWE's reputation, impact and relevance. Clearly, metrics are not the endgame. Publication and discoverability of science and its applications are the primary targets. Discoverability is aided by internal AGU efforts such as press releases and editor highlights and by broader efforts in indexing. In particular, beyond inclusion in Web of Science and SCOPUS, SWE is now indexed in IEEE Xplore at http://ieeexplore.ieee.org/search/advsearch.jsp?expression-builder and also captured in the new beta version for the Astrophysics Data System (ADS), https://ui.adsabs.harvard.edu.

This good news is balanced with some concerns. Recently AGU sent a survey on Centennial Grand Challenges to the AGU College of Fellows. The few anonymous replies for Space Weather seemed like a collective shoulder-shrug. We feel strongly that AGU's senior brain trust could have done a better job in answering the mail. Space weather does have grand challenges and the SWE editorial team contributed a number of ideas after seeing the lack-luster replies.

We invite active SWE authors to contribute to advancing the journal's impact: 1) Write your three main points to highlight your breakthrough science—statements of the obvious are not very impactful; 2) Cite relevant literature previously published in SWE_-after 14 years there is a critical mass knowledge; 3 ) When submitting manuscripts, include a plain language summary. While journal metrics likely don't keep editors up at night, writing editor's highlights do. Help out your editors (and yourselves) by putting your results into a form that helps us easily showcase your work; 4) Finally, answer review requests promptly. If you are too busy, let the requesting editor know immediately and recommend other reviewers. Doing so keeps our turn-around in the one-month range; 5) If you see a SWE manuscript highlighted beyond the bounds of Altmetric and scientific journal citations let the editorial team know. These few simple acts make a big difference to the SWE editorial team and allow us to keep SWE at the forefront.

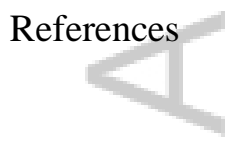


Aplin, K. L., A. A. Briggs, R. G. Harrison, and G. J. Marlton (2017), Measuring ionizing radiation in the atmosphere with a new balloon-borne detector, Space Weather, 15, 663-672,

doi:10.1002/2017SW001610.

Molera Calvés, G., Kallio, E., Cimo, G., Quick, J., Duev, D. A., Bocanegra Bahamón, T., ... Mikhailov, A. (2017). Analysis of an interplanetary coronal mass ejection by a spacecraft radio signal: A case study. Space Weather, 15. https://doi.org/10.1002/2017SW001701

Dachev, T. P., Bankov, N. G., Tomov, B. T., Matviichuk, Y. N., Dimitrov, P. G., Häder, D.-P., \& Horneck, G. (2017). Overview of the ISS radiation environment observed during the ESA EXPOSE-R2 mission in 2014-2016. Space Weather, 15. https://doi.org/10.1002/2016SW001580

Harrison, R. A., J. A. Davies, D. Biesecker, and M. Gibbs (2017), The application of heliospheric imaging to space weather operations: Lessons learned from published studies, Space Weather, 15, 9851003, doi:10.1002/2017SW001633.

Kelly, M. A., J. M. Comberiate, E. S. Miller, and L. J. Paxton (2014), Progress toward forecasting of space weather effects on UHF SATCOM after Operation Anaconda, Space Weather, 12, 601-611, doi: 10.1002/2014SW001081.

Lanzerotti, L. J. (2007), Space Weather Receives First Impact Rating. Space Weather, 5: n/a.

doi:10.1029/2007SW000353

$\geqslant$

Morley, S. K., J. P. Sullivan, M. R. Carver, R. M. Kippen, R. H. W. Friedel, G. D. Reeves, and M. G. Henderson(2017), Energetic Particle Data From the Global Positioning System Constellation, Space Weather, 15, 283-289, doi:10.1002/2017SW001604.

Sharpe, M. A., \& Murray, S. A. (2017). Verification of space weather forecasts issued by the Met Office Space Weather Operations Centre. Space Weather, 15, 1383

1395. https://doi.org/10.1002/2017SW001683

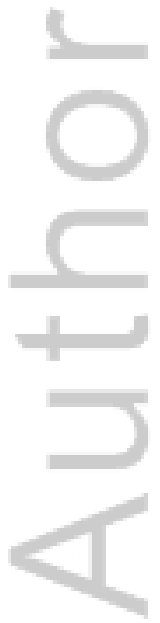

